VeritasE Scientia

Vol. 8. $\mathrm{N}^{\circ} 1$.

Enero - Junio del 2019.

ISSN Edición Online: 2617-0639

https://doi.org/10.47796/ves.v8i1.109

\title{
JUSTICIA EN EL DERECHO PRIVADO Y RESPONSABILIDAD CIVIL EXTRACONTRACTUAL POR DAÑO NO PATRIMONIAL EN LA JURISPRUDENCIA PERUANA
}

\author{
JUSTICE IN PRIVATE LAW AND EXTRACONTRACTUAL CIVIL LIABILITY FOR NON-PATRIMONIAL \\ DAMAGE IN PERUVIAN JURISPRUDENCE
}

Enlil Iván Herrera Pérez ${ }^{1}$

Dennis José Almanza Torres ${ }^{2}$

Presentado: 09/11/2018

Aceptado: $27 / 11 / 2018$

Publicado online:17/07/2019

\section{RESUMEN}

¿Qué es lo que busca el Derecho de daños a través de la responsabilidad civil? ¿Disuadir una conducta injusta? ¿Castigar al agente dañador? ¿Indemnizar a la víctima del daño? Las respuestas pueden ser diferentes de acuerdo a cada punto de vista, y sin embargo nos preguntamos ¿̇y qué es lo "justo" en la responsabilidad civil? Bajo esa motivación intentamos responder la siguiente inquietud en la presente investigación: ¿Se aplica una forma de justicia adecuada al Derecho Privado en los casos de responsabilidad civil extracontractual por daño no patrimonial en la jurisprudencia peruana? Y para tal efecto (i) desarrollamos un marco filosófico sobre la justicia en el ámbito señalado, (ii) describimos el desarrollo doctrinal y normativo referente al daño no patrimonial, y (iii) analizamos los criterios empleados en la jurisprudencia peruana. Así, a través de un método de interpretación sistemática y dogmática, llegamos a una preocupante conclusión: no se aplica una forma de justicia adecuada.

Palabras clave: Derecho privado, Derecho de daños, justicia, daño no patrimonial, jurisprudencia.

\begin{abstract}
What does Tort Law seek through civil liability? Just deterrence? Punish the tortfeasor? Compensate the victim? The answers may be different according to certainly point of view, nevertheless we asked ourselves: what is "fair" in the civil liability context? Under that motivation, we tried to answer the following questions: is the way of justice suitable as is applied in non-economic loss cases in the Peruvian case law? And for that purpose, (i) we developed a philosophical framework about justices in this context, (ii) we described the legal doctrine and regulation framework, and (iii) we
\end{abstract}

\footnotetext{
${ }^{1}$ Maestro en Derecho con mención en derecho civil y comercial

${ }^{2}$ Doctor, Académico de la Escuela de Postgrado de la Universidad Privada de Tacna
} 
analyzed the criteria adopted in the Peruvian case law. Thus, through a systematic and dogmatic methods of interpretation, we reached a worrying conclusion: a suitable way of justice is not applied in Peruvian case law of non-economic loss in the context of Tort Law.

Key words: Private law, tort law, justice, non-economic loss, case law.

\section{INTRODUCCIÓN}

Sin duda alguna el Derecho implica un concepto "multívoco", lo que supone que tiene diversos conceptos y que ninguno es el "único correcto" y al mismo tiempo que ninguno es "falso", sin embargo, existe una distinción en considerar a los componentes del Derecho como meta-componentes, e intentar explicar sus instituciones dotándolas de una "naturaleza", o en considerarlos como meras herramientas para un fin que los operadores determinen y estudiar su eficiencia, implicando en ambos casos estudiar al Derecho y componentes como lo que son y/o como se ven, y -por otro lado- en considerar a los componentes del Derecho, además, como deben ser, esto es, buscando su orientación valorativa, que en última instancia supone el anhelo de lograr justicia.

El Derecho Privado no es ajeno a ello, sino que, a través de sus diversas instituciones, cada una con sus particularidades, también "camina" en el mismo "rumbo". Así, dentro de este campo tenemos al Derecho de daños, el cual se cimienta sobre aquello conocido como Responsabilidad Civil, y aunque el "rumbo" sea el mismo, preocupa saber cómo va y cómo debe ir en dicho "rumbo". Sin embargo, nuestra jurisprudencia parece no "despejar" dicha preocupación, sino incluso "agravarla", y es por ello que el objetivo principal de la presente investigación consiste en demostrar que la forma de justicia aplicada en los casos de responsabilidad civil extracontractual por daño no patrimonial en la jurisprudencia peruana bajo un criterio distributivo no es adecuada a la naturaleza del Derecho privado. Ciertamente, hablar sobre el "daño" en el Derecho resulta una cuestión "compleja", pero que no debe pasar inadvertida en el debate jurídico; distintos tópicos, desde la consideración propia del "daño" como tal, o la cuantificación de daños no patrimoniales, y hasta incluso aspectos filosóficos y discusiones sobre la "justicia" en este contexto. Ahora bien, ¿qué contexto? ¿Con qué propósito o propósitos? ¿Cómo hablar en términos de "justicia"? La responsabilidad civil extracontractual es uno de los pilares del Derecho Privado, entonces ese es nuestro contexto. Ahora bien ¿qué propósito puede tener la responsabilidad civil extracontractual en dicho contexto? La doctrina italiana tradicional dice que incluso existe una función punitiva y por ende también una disuasiva ¿encaja ello con el fundamento y lo característico del Derecho Privado, o estaremos desnaturalizando instituciones jurídicas? Y aunque ciertamente el propio concepto de "Derecho Privado" puede ser variado, el formalismo jurídico aporta uno sobre la base de la coherencia justificativa que se centra en las particularidades y unidad de las figuras e instituciones jurídicas en determinado contexto, así, el Derecho Público también tendrá su propia caracterización. En tal sentido, las manifestaciones de justicia dependen del ámbito de las relaciones jurídicas, así, para el caso de relaciones jurídico-privadas correspondería la aplicación de la denominada justicia correctiva o conmutativa, la cual postula en el ámbito de la responsabilidad civil extracontractual que, al ocurrir un daño se produce una "injusticia" o una "desigualdad", lo que amerita una "corrección" de lo sucedido para retornar a un estado de "igualdad" o "equilibrio".

Scott J. Shapiro (2010) se pregunta sobre el por qué debería importarnos saber qué es el Derecho, señalando: "The inquiry into the nature of law is not an exercise in lexicography, extends far beyond determining whether an unjust rule is a law and is more than of mere academic interest." En efecto, la compresión y armonización de las instituciones del Derecho no gira en torno a cuestiones formales, si bien 
el Derecho puede verse impulsado por una "teoría del caos", dicha "armonización", que además engloba lo que acontece en la práctica judicial, no debe ser vista como un "objetivo", sino como un "camino" a la propia justicia. En tal sentido, una incorrecta comprensión acerca de la naturaleza del Derecho privado, o del fundamento mismo de la responsabilidad civil, deforma la idea de qué tipo de justicia debe aplicarse en este particular ámbito del Derecho, y con una imagen nublada que busca "justicia" un operador del Derecho puede estar propiciando, al contrario, injusticia a través de arbitrariedad. Por ello, resulta necesaria una investigación una perspectiva distinta a los clásicos estudios analíticos y descriptivosemánticos o históricos realizados sobre la responsabilidad civil.

\section{El daño no patrimonial en la responsabilidad civil extracontractual en el Derecho peruano}

"Ilícito civil", un concepto cuya reconstrucción histórica y relación con el daño nos pone en la necesidad de su mención, pero que al mismo tiempo nos coloca en una situación problemática: el concepto del "ilícito civil" varía de acuerdo al tiempo, lugar y sujeto que lo esboza. ¿A qué se refieren los autores con "ilícito civil"? Las respuestas varían, situando al "ilícito civil" como un hecho, otros como un acto, unos proponiendo una estructura con un número determinado de elementos, otros con más y otros con menos. Guido Alpa (2016) advierte ello y señala: "De cualquier forma, el análisis de las fuentes históricas nos ofrece una ayuda valiosa en la comprensión de los términos, pero también nos imparte una lección que no debemos olvidar: las expresiones usadas, tales como hecho ilícito, daño injusto, culposo y similares, son matizados con significados que divergen según el contexto y el autor, y, por lo tanto, no constituyen una constelación de estrellas fijas." (pp. 150, 151)

Así, tendríamos expresiones con características más, características menos, que inicialmente nos situarían en el escenario de "las obligaciones que nacen de los delitos" -y cuasi-delitos- (Cfr. Grocio, Trad. 1925; Domat, Trad. 1841; Pothier, Trad. 1839), en este contexto, Guido Alpa (2016) señala que lo que se reputaría como "ilícito" sería el "hecho", sea por "culpa" o por "impericia", pero en todos los casos con un resultado dañoso a un tercero. Posterior a ello se introduce el concepto de "acto ilícito" (Cfr. Windscheid, 1904; De Ruggiero, 1931) sustentado en un acto activo u omisivo, en todo, un comportamiento. Introducimos un segundo concepto que es elemento del primero: el daño. Guido Alpa (2016) defiende una concepción ontológica, según la cual por daño entenderíamos la "lesión de un interés protegido", nótese que la postura de Alpa distingue entre "daño" y perjuicio o agravio (en inglés diríamos "harm"), una situación que guarda coherencia con los Principios de derecho europeo de la responsabilidad civil esbozados por el European Group on Tort Law (2005) el cual sustenta de igual manera que "El daño requiere un perjuicio material o inmaterial a un interés jurídicamente protegido", sin embargo, existe una segunda concepción más enfocada en las consecuencias que define al daño como "la alteración negativa de una determinada situación de la víctima, económica o incluso física o psíquica" (Salvi, 2015).

En todo, debe notarse cómo las escuelas de Derecho continental centran su visión en las "consecuencias relevantes" tales como que "la patrimonialidad es un requisito inherente al daño y no a la naturaleza del bien o interés lesionado" (Salvi, 2015, p. 275) incorporándose además un concepto de "patrimonio moral" (Salvi, 2015). El espacio de debate en Perú se encuentra abierto y puede considerarse al efecto la Casación № 01125-95-Arequipa (1998) que sostuvo como argumento el siguiente: "En nuestro sistema de responsabilidad civil, la reparación del daño moral debe abarcar el proveniente del incumplimiento de cualquier obligación, siendo así el interés del reclamante puede ser patrimonial o no, cuestión que no debe confundirse con el carácter pecuniario de la prestación, ya que la ley protege no solamente los intereses patrimoniales sino también los de naturaleza extrapatrimonial, sea que se cause perjuicios económicos o no" (p. 1389). Sobre lo acotado, resaltamos la última línea: "sea que cause perjuicios económicos o no", en tal sentido, podría argumentarse en Perú que es posible hablar de la corrección de pérdidas/ganancias (injustas) normativas. 
Las "consecuencias" derivadas del daño no patrimonial en el régimen de la responsabilidad civil extracontractual en el Código Civil peruano.

Por "consecuencias", ahora bien, entendemos aquellas de orden económico que se generan por los daños causados, en este caso, refiriéndonos específicamente en el contexto de los daños no-patrimoniales, "consecuencias" para Gastón Fernández Cruz (2015). Clarifica el panorama de esta situación y nos permite consolidar una idea: no siempre se puede "valuar" el daño en forma "objetiva" -salvo que se establezca una solución de "tablas" aunque de igual manera el fundamento de dichas tablas no escaparía de todo de una esfera subjetiva para su establecimiento. Esto nos lleva a la necesidad de diálogo sobre un punto teórico más, antes de analizar las tendencias jurisprudenciales.

La noción de justicia subyacente en los criterios de valoración del daño no patrimonial en los casos de responsabilidad civil extracontractual. Un análisis jurisprudencial

El hecho de que el juez sea un ser humano frente a una labor de dar solución a una controversia o esclarecer una situación con relevancia jurídica, supone que comparecen en él tres elementos indesligables (Valdivia Cano, 2017) para resolver dicho "problema": su cosmovisión personal, su concepción jurídica, y el problema como tal. Esto, sin embargo, no significa que en su "trabajo final" (es decir, la sentencia) se plasmen estos tres elementos en forma expresa y/o "detallada", pero concurren al verter su razonamiento como argumentos quizá en forma consciente o no. De ello es que tenemos la existencia de una "noción de justicia" que subyace a su razonamiento, o en otras palabras, que está "oculta" en las consideraciones jurídicas plasmadas, las que determinarán si el daño es o no resarcible, si existe o no causalidad y así también sobre las consecuencias económicas resultantes del daño, la cuantificación del mismo, reflejando así si se emplea un criterio resarcitorio o bien uno aflictivoconsolatorio o uno punitivo para tal efecto, buscando "corregir" el desequilibrio producido por el daño o "distribuir" los recursos a su criterio -que no necesariamente será uno igualitarista.

\section{OBJETIVOS}

a) Argumentar un concepto de justicia en el ámbito de la responsabilidad civil extracontractual.

b) Analizar la regulación del daño no patrimonial en el ámbito de la responsabilidad civil extracontractual en el Código Civil peruano.

\section{METODOLOGÍA}

Investigación básica o pura, documental y dogmática, puesto que se pretende el desarrollo de interpretación y argumentación en relación a instituciones y categorías jurídicas a la luz de normas jurídicas y doctrina de la materia. El nivel es exploratorio, con un carácter argumentativo. La investigación ha sido realizada en la ciudad, provincia y departamento de Tacna y abarca una dimensión temporal contemporánea, haciendo referencia a jurisprudencia (case law) peruana desde el año 1995 a la fecha. Se ha delimitado deliberadamente el número de elementos contenidos en la población descrita, empleando un método de muestreo no aleatorio o no probabilístico, incidiendo en caso de la jurisprudencia, sobre sentencias de responsabilidad civil extracontractual por daño no patrimonial. En el caso de tales últimas el criterio discriminador se ha realizado en función de seleccionar sentencias que declaren y cuantifiquen daños no patrimoniales en el contexto de responsabilidad civil extracontractual en materia de Derecho Civil, excluyéndose aquellos que impliquen sentencias infundadas o que declaren la improcedencia de pretensiones, así también excluyéndose aquellos discutidos en otras vías, sea penal, laboral o familiar. 


\section{RESULTADOS}

«Un concepto de justicia coherente y satisfactorio conforme al Derecho Privado y, por ende, en el ámbito de la responsabilidad civil extracontractual, es el propuesto por la justicia correctiva o conmutativa.» La citada hipótesis es desarrollada en el primer sub-capítulo del marco teórico el cual se denomina "Hacia un concepto de justicia en el ámbito de la responsabilidad civil extracontractual", considerando que el objetivo específico ligado a esta hipótesis era el de justamente argumentar un concepto de justicia en el ámbito demarcado.

Al mismo tiempo, dicho sub-capítulo constituye el marco filosófico que dota de consistencia deontológica para argumentar la hipótesis citada, en tal sentido, hemos observado que existen diversas posturas en relación a la responsabilidad civil extracontractual, así, tenemos a las teorías instrumentalistas y las no instrumentalistas, dentro de las primeras hemos analizado a lo propuesto por el Law and Economics, sin embargo, al tratar únicamente a la responsabilidad extracontractual como mero instrumento, sin un propósito propio que tenga pues para el Law and Economics la justicia no es un objetivo medible en términos de eficacia, vacía de contenido a la institución, teniendo incluso dificultades en su concepción si sólo se la trata únicamente como instrumento de eficiencia, aunque no pueda negarse la utilidad del Análisis Económico. Por otro lado, también dentro de las teorías instrumentalistas, tenemos teorías morales que buscan "prescribir" y otras "remediar" a través de sus postulados, considerando principalmente a la teoría de la justicia distributiva, así como a su vertiente igualitarista, sin embargo, la misma también presenta dificultades en su aplicación, aunque no pueda negarse la importancia valorativa de la misma, dificultades que se presentan al intentar aplicarla en el contexto de aquello característico del Derecho Privado: las relaciones bilaterales entre privados, horizontales, no de administradoradministrados; y que supone aún un inconveniente mayor: el enriquecimiento injusto.

Finalmente, hemos analizado una teoría no instrumentalista, que también es parte de las teorías morales, denominada "formalismo jurídico", mediante la cual el Derecho de daños podría explicarse como tal, con un contenido propio y específicamente dirigido a como está diseñado el Derecho Privado, en tal sentido, considerando como uno de los elementos centrales en lo que es la autonomía de las personas, su libertad de actuar y de regularse, así como su deber de no causar daño y habiendo causado el mismo de repararlo, tenemos que una justicia que puede actuar sobre las relaciones privadas bilaterales frente a una ganancia/pérdida injusta que invade la esfera de la autonomía de la persona y que puede reparar dicha injusticia es la justicia correctiva.

"La regulación en el Código Civil peruano del daño no patrimonial en el ámbito de la responsabilidad civil extracontractual adolece de defectos que afectan la claridad de su comprensión dejan un margen de interpretación amplio al no delimitar las consecuencias por daño no patrimonial y los criterios de cuantificación del mismo.»

Esta hipótesis es argumentada en base a como "El daño no patrimonial en la responsabilidad civil extracontractual en el Derecho peruano", en el cual hemos examinado la concepción del daño en la doctrina, tanto extrajera como nacional, de lo general a lo específico, comentando así mismo sobre la codificación y regulación del daño no patrimonial actual. Así, se ha percibido que la actual regulación de lo referido ha tenido un proceso de codificación informal e irregular, introduciéndose voces como "daño a la persona" y a la par "daño moral" sin mayor análisis, y aunque existan excelentes investigaciones -tal como hemos visto- que permiten "reinterpretar" en forma armónica lo regulado mediante la reclasificación del daño, esta visión integradora no es muchas veces captada en la jurisprudencia, tal como se aprecia en el tercer sub-capítulo. Pero lo más preocupante es la consideración en la norma de conceptos ambiguos y figuras complejas como el daño no patrimonial, una preocupación que se refleja en lo normado para su cuantificación: la "equidad". Así, hemos visto a autores que expresan dicha preocupación en términos de la posibilidad de "círculos viciosos" así como del riesgo de enriquecimiento 
indebido, producto de una falta de regulación más concreta de aquellos criterios que debe tener en cuenta cada juez para resolver, preocupación que se torna en realidad al contemplar lo expuesto en el tercer sub-capítulo sobre el desarrollo jurisprudencia.

«Los criterios de aplicación de justicia en la jurisprudencia peruana sobre casos de responsabilidad civil extracontractual por daño no patrimonial no se encuentran unificados, observándose casos en los que la cuantificación de los daños difiere en forma abismal, a pesar de ser casos con características similares, lo que refleja en la práctica un criterio subyacente de "justicia distributiva".»

La argumentación de esta hipótesis requirió comparar los criterios de aplicación de justicia en la jurisprudencia peruana con las posturas existentes y concepción de la justicia desarrollados en los subcapítulos primero y segundo, hipótesis que es desarrollada en el tercer sub-capítulo en la cual nos abocamos a la práctica judicial en casos que giran en torno al daño no patrimonial. En tal sentido, en primer lugar, hemos descrito los criterios de aplicación de justicia a través de 6 casos ejemplares en los que se resuelve a nivel de Corte Suprema y también de Corte Superior revocar el quantum indemnizatorio establecido en instancias inferiores por el concepto de daño no patrimonial y modificarlo en atención a una "fundamentación" que en la mayoría de casos citados se encuentra ausente o resulta completamente insuficiente. En segundo lugar, hemos ordenado los criterios sostenidos en diversa jurisprudencia (case law) de acuerdo a las funciones de la responsabilidad civil, así como también de acuerdo a tipos de casos en los que concurre siempre pretensión por daño no patrimonial.

«En la jurisprudencia peruana, en los casos de responsabilidad civil extracontractual por daño no patrimonial, no se aplica una forma de justicia adecuada al Derecho Privado.»

Llegamos a esta hipótesis a través del desarrollo argumentativo, y de contrastar lo abordado en el marco filosófico, con la doctrina enfocada en la responsabilidad civil extracontractual como institución del Derecho Privado, y con la práctica judicial (case law) sobre casos de responsabilidad civil por daño no patrimonial. Así, habiendo sustentado debidamente que la expresión de justicia más apegada al Derecho privado, considerando su estructura, es la justicia correctiva; habiendo argumentado así también que la regulación actual en la legislación peruana sobre el daño no patrimonial en el contexto de la responsabilidad civil extracontractual adolece de defectos que afectan su claridad y comprensión, dejando un espacio de discrecionalidad a los jueces y no orientando algún criterio previsible para la concepción del daño como para su cuantificación; y habiendo sostenido que el criterio de justicia subyacente en la práctica judicial sobre casos de daño no patrimonial no es unitario, pero que en su diversidad mayoritariamente se desprende un criterio distributivo; es que, podemos percibir que estamos frente a una situación de "caos" en lo analizado, y por más que se argumente incluso a nivel jurisprudencial en favor de un criterio correctivo, o de incluso uno igualitarista, lo que resulta en la práctica es un discurso que muchas veces no va unido con lo concluyente en las sentencias, o que en otros casos simplemente no llega a consolidarse como uno argumentativo suficiente. Entonces, considerando que la jurisprudencia peruana se apega a un criterio distributivo genérico, vemos materializada nuestra preocupación: En la jurisprudencia peruana, en los casos de responsabilidad civil extracontractual por daño no patrimonial, no se aplica una forma de justicia adecuada al Derecho Privado.

\section{DISCUSIÓN}

En la responsabilidad civil extracontractual, como uno de los pilares del Derecho privado, se ha argumentado la aplicación de diversas teorías de la justicia tales como la correctiva o la distributiva, y aunque esta última resulte persuasiva debido a las propuestas valorativas y sociales, es la justicia correctiva la expresión más adecuada frente a relaciones sustantivas de Derecho privado (agente dañador

- víctima) en las que media una conducta indeseable (ganancia/pérdida injusta), y que -inter alia- 
ameritan una solución igualmente bilateral (corrección de la ganancia/pérdida injusta), elementos que sólo son considerados en forma integral (y a un mismo tiempo) a través de la justicia correctiva.

La doctrina, con un rol trascendental orientando la construcción e interpretación del Derecho es rica y diversas incluso para el Derecho de daños, implicando diferentes (e incluso distantes) posturas, lo que, sumado a una regulación normativa inexacta, laxa o ambigua, puede devenir en una falta de unidad interpretativa, situación que sucede en nuestro ordenamiento jurídico en relación a la misma concepción del daño y a la cuantificación de tal, propiciando un margen de discrecionalidad amplio en los juzgadores y afectando así previsibilidad jurisprudencial (case law) -en sentido estricto.

En la jurisprudencia peruana (case law) no se percibe unidad de criterio sobre casos de daño no patrimonial, sino diversas posiciones doctrinarias asumidas, así como criterios valorativos sobre el daño, que permiten afirmar la existencia de una margen de discrecionalidad amplio en la práctica judicial. Sin embargo, aún con dicha multiplicidad de criterios, el criterio que subyace a lo que finalmente se decide sobre el daño y a la cuantificación del mismo, dista de acercarse a lo postulado por la justicia correctiva, y en muchos casos, incluso dista de lo propuesto por las teorías morales del Derecho.

Teniendo en panorama el desarrollo normativo y jurisprudencial peruano sobre el daño no patrimonial en el contexto de la responsabilidad civil extracontractual, podemos afirmar que no se aplica una forma de justicia adecuada. Pero también podemos sostener algo que resulta más preocupante aún: la falta de precisión normativa y de orientación valorativa (value-oriented), sumado a la insuficiencia argumentativa sobre la concepción y cuantificación del daño, deja a un amplio margen de potencial arbitrariedad del juzgador este tipo de casos, impidiendo, además, la previsibilidad de criterios jurisprudenciales.

Se recomienda establecer un sistema de tablas para la determinación del quantum indemnizatorio en los diferentes casos, y concibiendo asimismo factores agravantes y atenuantes sobre lo establecido. Como segundo punto se recomienda mejorar el sistema de jurisprudencia (case law) peruano, ya que, en la actualidad no permite una visualización panorámica de los criterios existentes o mayoritarios, así, esta recomendación se dirige a la implementación y mejora de dicho sistema tanto a nivel de Corte Suprema como de Cortes Superiores. Además de permitir una visualización panorámica de criterios existentes, ello posibilitaría una herramienta útil a los mismos magistrados, así como servir de igual manera de herramienta a los litigantes y a los justiciables en general, propiciando al mismo tiempo la posibilidad de transparencia y "fiscalización" de lo que se ordena.

\section{REFERENCIAS BIBLIOGRÁFICAS}

Alcorn v. Mitchell, 63 III. 553 (Supreme Court of Illinois June 1872).

Alpa, G. (2016). La responsabilidad civil. Lima: Ediciones Legales.

Beltrán Pacheco, J. (2010). Valorización equitativa del resarcimiento. In G. Jurídica, Código Civil Comentado (Tercera ed., Vol. VI, pp. 739-741). Lima: Gaceta Jurídica.

Black, H. C. (1979). Black's Law Dictionary. St. Paul Minn.: West Publishing Co.

Borghetti, J.-S. (2014). Los intereses tutelables y la dimensión de los perjuicios reparables en el Derecho Francés de la Responsabilidad Civil Extracontractual. Thēmis - Revista de Derecho, Nro. 66, 285307.

Bullard González, A., Escobar Rozas, F., \& León Hilario, L. (2012, Diciembre). Temas de responsabilidad civil en debate: nuevas tendencias. lus et Veritas, Nro. 45, 422-433.

Calabresi, G. (1970). The Cost of Accidents: A Legal and Economic Analysis. New Haven: Yale University Press.

Calabresi, G. (1975). Concerning Cause and the Law of Torts: An Essay for Harry Kalven, Jr. The University of Chicago Law Review, 43(1), 69-108. 
Calabresi, G. (2016, Julio). Una visión más amplia de la Catedral: El significado de la regla de responsabilidad. Corrigiendo un malentendido. lus et Veritas, Nro. 52, 384-396.

Calabresi, G., \& Melamed, A. D. (1996). Reglas de propiedad, reglas de responsabilidad y de inalienabilidad: Una vista de la catedral. Estudios Públicos, 63, 347-391.

Cane, P. (2006). Atiyah's Accidents, Compensation and the Law. Cambridge: Cambridge University Press.

Cappelletti, M. (2015). Punitive Damages and the Public/Private Distinction: A Comparison Between the United States and Italy. Arizona Journal of International and Comparative Law, 32(3), 799-848.

Casación N. 3289-2015-Callao (Corte Suprema de Justicia de la República del Perú Enero 19, 2017).

Casación № 00579-2005-Lima (Corte Suprema de Justicia de la República del Perú Diciembre 20, 2005).

Casación № 00635-2012-Lima (Corte Suprema de Justicia de la República del Perú Noviembre 15, 2012).

Casación № 00731-2015-La Libertad (Corte Suprema de Justicia de la República del Perú Noviembre 27, 2015).

Casación № 01125-95-Arequipa (Corte Suprema de Justicia de la República del Perú Mayo 22, 1998).

Casación № 02108-2014-Lima (Corte Suprema de Justicia de la República del Perú Julio 13, 2015).

Casación № 0785-2015-Huánuco (Corte Suprema de Justicia de la República del Perú Noviembre 26, 2015).

Casación № 1363-2014-Huancavelica (Corte Suprema de Justicia de la República del Perú Mayo 22, 2015).

Casación № 1676-2004-Lima (Corte Suprema de Justicia de la República del Perú Setiembre 26, 2005).

Casación № 4393-2013-La Libertad (Corte Suprema de Justicia de la República del Perú Junio 19, 2014).

Casación № 5721-2011-Lima (Corte Suprema de Justicia de la República del Perú Julio 2, 2013).

Cassell and Co. Ltd. v. Broome and another, [1972] UKHL 3 (House of Lords February 23, 1972).

Chapman, B. (2002). Pluralism in Tort and Accident Law: Towards a Reasonable Accommodation. In G. J.

Postema, Philosophy and the Law of Torts (pp. 276-321). Cambridge: Cambridge University Press.

Chiassoni, P. (2013). El Análisis Económico del Derecho. Lima: Palestra Editores.

Coleman, J. L. (1988). The Economic Structure of Tort Law. Faculty Scholarship Series, 97, 1233-1253.

Coleman, J. L. (1992). Risks and Wrongs. Cambridge: Cambridge University Press.

Coleman, J. L. (2010). Riesgos y Daños. Madrid: Marcial Pons.

Cooter, R., \& Ulen, T. (2011). Law and Economics (Sixth ed.). Boston: Pearson.

Costanza, M. (2003). Qualche riflessione sul danno esistenziale a margine di una sentenza del Tribunale di Milano. Giurisprudenza Italiana, Anno CLV(IV), 1063-1064.

De Trazegnies Granda, F. (1980). Anteproyecto de la Comisión Reformadora del Código Civil. Lima.

De Trazegnies Granda, F. (2001). La responsabilidad extracontractual (Sétima ed., Vol. II). Lima: Fondo Editorial de la Pontificia Universidad Católica del Perú.

Dimond v. Lovell, [2000] UKHL 27 (House of Lords Mayo 11, 2000).

Englard, I. (2009). Corrective \& Distributive Justice: from Aristotle to modern times. New York: Oxford University Press.

Espinoza Espinoza, J. (2016). Derecho de la Responsabilidad Civil (Octava ed.). Lima: Instituto Pacífico.

European Group on Tort Law. (2005). Principios de derecho europeo de la responsabilidad civil.

Fernández Cruz, G. (2015). La dimensión omnicomprensiva del daño no patrimonial y la reclasificación de los daños. In J. (. Espinoza Espinoza, Análisis sistemático del Código Civil: A tres décadas de su promulgación (pp. 489-524). Lima: Instituto Pacífico.

Ferrer Beltrán, J., Moreso, J. J., \& Papayannis, D. M. (Eds.). (2012). Neutralidad y teoría del derecho. Madrid: Marcial Pons.

Franzoni, M. (1999). La evolución de la responsabilidad a partir del análisis de sus funciones. lus et Veritas, Nro. $18,68-87$.

Franzoni, M. (1999). La función del resarcimiento del daño no patrimonial. De lure, Año 1(1), 21 y ss.

Franzoni, M. (2010). Il danno risarcible (Seconda ed.). Milano: Giuffre.

Fried, C. (2015). Contract as promise: a theory of contractual obligation (Second ed.). New York: Oxford University Press. 
Galgano, F. (2010). Trattato di diritto civile (Vol. I). Milano: CEDAM.

Geistfeld, M. (2002). Economics, Moral Philosophy, and the Positive Analysis of Tort Law. In G. J. Postema, Philosophy and the Law of Torts (pp. 250-275). Cambridge: Cambridge University Press.

Gold, A. S. (2011). A moral rights theory of private law. William and Mary Law Review, 52(6), 1873-1931.

Goldberg, J. C. (2011). Tort in Three Dimensions. Pepperdine Law Review, 38(2), 321-336.

Goldberg, J. C. (2012). Introduction: Pragmatism and Private Law. Harvard Law Review, 125(7), 1640-1663.

Goldberg, J. C. (2015). Inexcusable Wrongs. California Law Review, 103(3), 467-512.

Goldberg, J. C., \& Zipursky, B. C. (2007). Tort Law and Moral Luck. Cornell Law Review, 92(6), 1123-1176.

Gómez Marín, M., \& Gil y Gómez, P. (Eds.). (1872). El Digesto del emperador Justiniano (Vol. I). (B. A. Rodríguez de Fonseca, Trans.) Madrid: Ramón Vicente.

Hegel, G. W. (1979). Sobre las maneras de tratar científicamente el Derecho Natural. (D. Negro Pavon, Trans.) Madrid: Aguilar.

Hershovitz, S. (2014). Tort as a Substitute for Revenge. In J. Oberdiek, Philosophical Foundations of The Law of Torts (pp. 86-102). Oxford: Oxford University Press.

Holmes, O. W. (1897). The Path of Law. Harvard Law Review, 10, 457-478.

Horwitz, M. J. (1982). History of the Public/Private Distinction. University of Pennsy/vania Law Review, 130(6), 1423-1428.

Huang, B. I. (2014). Surprisingly Punitive Damages. Virginia Law Review, 100(5), 1027-1059.

Jackson, B. (1988). Law, Fact, and Narrative Coherence. Liverpool: Deborah Charles Publications.

Kant, I. (trad. 2008). La Metafísica de las Costumbres (Cuarta ed.). (A. Cortina Orts, \& J. Conill Sancho, Trans.) Madrid: Tecnos.

Keating, G. (2002). A Social Contract Conception of the Tort Law of Accidents. In G. J. Postema, Philosophy and the Law of Torts (pp. 22-71). Cambridge: Cambridge University Press.

Kelsen, H. (1957). What Is Justice? Justice, Law, and Politics in the Mirror of Science. Berkeley: University of California Press.

Keren-Paz, T. (2007). Torts, Egalitarianism and Distributive Justice. Hampshire: Ashgate Publishing.

Kiam v. MGN Ltd., [2002] EWCA Civ 43 (England and Wales Court of Appeal (Civil Division) January 28, 2002).

Lagden v O'Connor, [2003] UKHL 64 (House of Lords December 4, 2003).

Landes, W. M., \& Posner, R. A. (1987). The Economic Structure of Tort Law. Cambridge: Harvard University Press.

León Hilario, L. (2017). La responsabilidad civil: Líneas fundamentales y nuevas perspectivas (Tercera ed.). Lima: Instituto Pacífico.

MacCormick, N. (1984). Coherence in Legal Justification. In A. Peczenik, L. Lindahl, \& B. V. Roermund (Eds.), Theory of Legal Science (Vol. 176). Dordrecht: D, Reidel Publising Company.

Markel, D. (2009). How should punitive damages work? University of Pennsy/vania Law Review, 157(5), 1383-1484.

Merino Acuña, R. (2016). Justicia social y economía en la Teoría del Derecho. Lima: Palestra Editores.

Olson, T. B., Boutrous, T. J., \& Corboy, P. H. (1991). At Issue: Punitive Damages. ABA Journal, 77(1), 40-41.

Papayannis, D. M. (2010). Comprensión y justificación de la responsabilidad extracontractual. Tesis Doctoral. Barcelona: Universitat Pompeu Fabra.

Papayannis, D. M. (Ed.). (2013). Derecho de daños, principios morales y justicia social. Madrid: Marcial Pons.

Papayannis, D. M. (2016). El derecho privado como cuestión pública. Bogotá: Universidad Externado de Colombia.

Polinsky, M. A., \& Shavell, S. (1998, February). Punitive Damages: An Economic Analysis. Harvard Law Review, 111(4), 869-962.

Posner, R. A. (1970). Book Review (reviewing Guido Calabresi, The Cost of Accidents: A Legal and Economic Analysis [1970]). Chicago Law Review, 37, 636-648. 
Posner, R. A. (1986). Economic Analysis of Law (Third ed.). Boston: Little Brown.

Posner, R. A. (1987). The Decline of Law as an Autonomous Discipline: 1962-1987.pdf. Harvard Law Review, 100, 761-780.

Posner, R. A. (1997). Law and Legal Theory in England and America. Oxford: Clarendon Press.

Posner, R. A. (2005). Guido Calabresi's 'The Costs of Accidents': A Reassessment. Maryland Law Review, $64,12-23$.

Postema, G. J. (2002). Introduction: Search for an Explanatory Theory of Torts. In G. J. Postema, Philosophy and the Law of Torts (pp. 1-21). Cambridge: Cambridge University Press.

R.N. № 00449-2009-Lima (Corte Suprema de Justicia de la República del Perú Julio 9, 2009).

R.N. № 01658-2014-Lima (Corte Suprema de Justicia de la República del Perú Marzo 15, 2016).

Rawls, J. (2006). Teoría de la Justicia. México: Fondo de Cultura Económica.

Revoredo Marsano, D. (2015). Exposición de Motivos y Comentarios del Código Civil (Segunda ed., Vol. VI). Lima: Thomson Reuters.

Rookes v. Barnard, [1964] UKHL 1 (House of Lords January 21, 1964).

Salvi, C. (2015). El daño. En L. León, Estudios sobre la Responsabilidad Civil (pp. 268-298). Lima: ARA Editores.

Shapiro, S. J. (2010). What is law (and why should we care)? 1st. Conference on philosophy and law neutrality and theory of law. Girona: Marcial Pons.

Shavell, S. (2007). Economic Analysis of Accident Law. Cambridge: Harvard University Press.

Solarte Rodríguez, A. (2015). Responsabilidad civil y equidad en el Código Civil peruano de 1984. Análisis desde la perspectiva del Derecho comparado. In Derecho Civil Extrapatrimonial y Responsabilidad Civil (pp. 271-306). Lima: Gaceta Jurídica.

Soto Coaguila, C. A. (2015). Tratado de responsabilidad civil contractual y extracontractual: comentarios a las normas del código civil (Vol. I). Lima: Instituto Pacífico.

Soto Coaguila, C. A. (2015). Tratado de responsabilidad civil contractual y extracontractual: comentarios a las normas del código civil (Vol. II). Lima: Instituto Pacífico.

Stein, A. (2017, April). The domain of torts. Columbia Law Review, 117(3), 535-611.

Sunstein, C. R., Kahneman, D., \& Schkade, D. (1997). Assessing Punitive Damages... Chicago: Coase-Sandor Institute for Law \& Economics Working Paper No. 50.

Sunstein, C. R., Kahneman, D., Schkade, D., \& Ritov, I. (2001). Predictably Incoherent Judgments. Chicago: John M. Olin Program in L. \& Econ. Working Paper No. 131.

Sunstein, C. R., Schakde, D., \& Kahneman, D. (2000). Deliberating about Dollars: The Severity Shift. Columbia Law Review, 100(4), 1139-1175.

Sunstein, C. R., Schkade, D., \& Kahneman, D. (1999). Do People Want Optimal Deterrence? Chicago: John M. Olin Program in Law \& Economics Working Paper No. 77.

Tarello, G. (2017). El realismo jurídico americano. Lima: Palestra Editores.

Unger, R. M. (1983). The Critical Legal Studies Movement. Harvard Law Review, 96(3), 561-675.

$\checkmark$ Pleno Jurisdiccional Supremo en materia Laboral y Previsional (Corte Suprema de Justicia de la República del Perú Agosto 4, 2017).

Valdivia Cano, J. C. (2017). La Caja de Herramientas. Investigación jurídica integral. Arequipa: Fondo Editorial Francisco Mostajo.

Velasco Caballero, F. (2014). Derecho público más Derecho privado. Madrid: Marcial Pons.

Weinrib, E. J. (1989). Understanding Tort Law. Valparaiso University Law Review, 23(3), 485-526.

Weinrib, E. J. (2012). Corrective Justice. Oxford: Oxford University Press.

Weinrib, E. J. (2017). La idea de derecho privado. Madrid: Marcial Pons. 